\title{
Genome-Wide Identification and Expression Analysis of Flavonoid 3'-Hydroxylase Gene Family in Carthamus Tinctorius $\mathrm{L}$.
}

Hoàng Việt Nguyễn Quốc ( $\square$ harryken090495@gmail.com )

Jilin Agricultural University https://orcid.org/0000-0001-7820-2735

Kong Jie

Jilin Agricultural University

Naveed Ahmad

Jilin Agricultural University

Yang Zhuoda

Jilin Agricultural University

Wang Nan

Jilin Agricultural University

Ma Xintong

Jilin Agricultural University

Zhang Xin Yue

Jilin Agricultural University

Liu Xiuming

Jilin Agricultural University

Yao $\mathrm{Na}$

Jilin Agricultural University

Jin Libo

Jilin Agricultural University

\section{Research Article}

Keywords: Flavonoid 3'-hydroxylase (F3'H), safflower, expression analysis, subcellular localization

Posted Date: June 8th, 2021

DOI: https://doi.org/10.21203/rs.3.rs-575863/v1

License: (1) (1) This work is licensed under a Creative Commons Attribution 4.0 International License.

Read Full License 


\section{Abstract}

Objective

Through experiments and bioinformatic analysis clearly demonstrate considerable information about the genetic evolution of the flavonoid 3'-hydroxylase $\left(\mathrm{F}^{\prime} \mathrm{H}\right)$ gene in Safflower and in plants.

\section{Results}

Here, we performed genome wide survey of safflower genome and identified a total of $22 \mathrm{CtF}{ }^{\prime} \mathrm{H}$ enzyme encoding genes. Phylogenetic analyses revealed the classifications of these CtF3'Hs into nine subgroups demonstrating their evolutionary relationship. The distribution of the conserved protein motifs, and cisregulatory units of CtF3'Hs indicated essential structure-to-function components leading to the final function of protein or its interactions. Furthermore, the results of digital expression analysis and the qRTPCR pattern of 22 putative $\mathrm{CtF}^{\prime} \mathrm{H}$ genes during different flowering stages suggested their requisite roles in safflower petal pigmentation. In addition, the fusions construct of plant expression vector pCAMBIA1302-GFP-CtF3'H5 in onion epidermal cells verified the subcellular localization of CtF3'H5 to the plasma membrane. Subsequently, the prokaryotic expression and western blot hybridization of CtF3'H5 resulted in a stable $50.3 \mathrm{kD}$ target protein. These results partly demonstrate the influence of F3'Hs on plants.

\section{Conclusions}

In this study, the results of digital expression and qRT-PCR analysis of 22 putative CtF3'H genes in different flowering stages indicate their essential role in safflower petal pigmentation. clearly demonstrates significant information on the genetic evolution of important enzyme-coding genes and will provide a pathway for future functional studies of F3'Hs in safflower as well as in plants.

\section{Introduction}

Safflower (Carthamus tinctorius $L$.) is widely known as one of the important medicinal plants worldwide. Safflower contains various active ingredients, including flavonoids, quinolones, alkaloids, and safflower polysaccharides (Ambreen et al. 2018). Safflower can exert various biological effects, including antioxidant, anti-inflammatory (Alaiye et al. 2020), and antibacterial activities (Cho et al. 2017). It has been reported that safflower is beneficial for improving acute cerebral infarction and ischemic stroke (Wang et al. 2019). Among flavonoids, Safflower Yellow is the main active ingredient of safflower, and it has a variety of pharmacological effects such as dilating blood vessels, protecting myocardial ischemia, and antioxidant activity. Flavonoids are an important class of natural products(Cerqueira et al. 2021); In particular, they belong to the polyphenolic group of plant metabolites, which are widely found in fruits, vegetables, and some beverages. The biochemical effects as well as antioxidant abilities of flavonoids are directly related to various health diseases such as cancer, alzheimer's disease (AD)(Du et al. 2021), atherosclerosis, etc. They are also sometimes act as strong inhibitors of different group of enzymes, for 
example xanthine oxyase (XO)(Lin et al. 2021), cyclo-oxyase (COX), lipoxygenase, and 3-kinase phosphoinositide (Panche et al. 2016).

The flavonoid 3'-hydroxylase $\left(\mathrm{F} 3^{\prime} \mathrm{H}\right)$ is the core enzyme of flavonoid metabolic pathway which belongs to the superfamily of cytochrome P450 (Choudhary et al. 2016). F3'H hydroxylation of position 3 'of the B ring of flavonoids (Guo et al. 2019) directs the production of cyanide red pigments. The F3'H gene that encodes CYPs has been cloned and well investigated in a variety of plant species such as hybrid Petunia (De Palma et al. 2014), and Arabidopsis thaliana (Gao et al. 2020). Furthermore, it was also suggested that the precursors molecules of flavonols are usually hydroxylated using 3'-hydroxylation via the overexpression of SbF3'H in sorghum. Similarly, the incredible loss of glycine max fuzz was achieved by viral-mediated silence of the F3' $\mathrm{H}$ gene (Nagamatsu et al. 2009). Morover, the results of ectopic expression level of the MdF3'H identified from Malus $\times$ domestica stimulated the accumulation pattern of anthocyanin ( $Y$ et al. 2010). Nonetheless, the biosynthetic pathway of flavonoids in the safflower is still unknown. It is reported in the literature that the late branches of the flavonoid biosynthetic pathway produce flavanols, flavonols, or anthocyanins, Therefore, it is important to fully understand and investigate the evolution and regulation of flavonoid 3'-hydroxylase $\left(\mathrm{F}^{\prime} \mathrm{H}\right)$ enzyme encoding genes which is of great significance for the downstream regulation of flavonoid content in safflower(Ren et al. 2021).

The purpose of the present study was aimed to present a complete overview of the genome wide identification of CtF3'H enzyme encoding subgene family in safflower and further study the evolutionary classification from their ancestars. This study also investigates several structural and functional dynamics of CtF3'H subfamily using multiple in silico predictions and molecular techniques. This reseasrch work in general highlights the groundwork for the identification of candidate $C t F 3^{\prime} H$ genes for future molecular breeding programs to produce new safflower varieties with rich anthocyanin content.

\section{Materials And Methods}

\section{Plant Materials, vectors and strains}

The safflower Jihong early-maturing variety was used experimental source in this study. The seeds were purchased from Xinjiang Honghuayuan Technology Co., Ltd. and planted in the experimental station of the Jilin Agricultural University. The petals of safflower bud, initial, flowering and fade stages were used for expression analysis. Agrobacterium tumefaciens strain EHA105, E. coli BL21, E. coli DH5a cells, prokaryotic expression vector (PET28a+-CtCYP81E8), subcellular localization vector pCAMBIA1302CtCYP81E8-GFP) were constructed and stored in a refrigerator at $-80^{\circ} \mathrm{C}$ with $75 \%$ glycerol until the next use.

\section{Identification of CtF3'H in safflower}

The genome of $\mathrm{JH} 1$ variety of Carthamus tinctorius was extracted from the online project of Jilin Agricultural University (PRJNA399628; filed on August 23, 2017) in the NCBI

(https://www.ncbi.nlm.nih.gov). BioEdit software was used to BLAST the putative CtF3'H in the genome 
of safflower. The identified $C t F 3^{\prime} H$ genes were further validated on the Pfam database (http://pfam.xfam.org) and the identified accessions were assembled for further analysis. The molecular weight $(\mathrm{MW})$ and isoelectric point $(\mathrm{pl})$ of each $\mathrm{CtF}^{\prime} \mathrm{H}$ protein was calculated using the ExPASy program (http://www.baoasy.org/). The amino acid sequence of each protein was aligned using DNAMAN software (Version 7; Lynnon Corporation, Quebec, Canada), using the default parameters.

\section{Phylogenetic Analysis}

The amino acid sequences of 22 CF3'H Proteins extracted from safflower genome were aligned with the help of Clustal W (2.0) package. Similarly, 136 members of the Arabidopsis F3'H family were extracted from the Arabidopsis Information Resources for comparartive analysis. A neighbour-joining evolutionary tree was constructed from these 22 safflower CF3'H Proteins and 136 Arabidopsis F3'H proteins using 1000 bootstrap method in the MEGA 5 software version 4.1 (http://www.megasoftware.net/) (Schrago et al. 2018). The organization and divergence of the CF3'H Proteins in safflower with the Arabidopsis F3'H was clustered together in various clans following their structural and functional similarities which classfied them into different subfamilies.

\section{Motif Elicitation and Promoter Analysis}

The conserved protein motifs of the CtF3'H proteins were determined by uploading 22 amino acid sequences into the MEME web server's online tool (http://meme.nbcr.net/meme/cgi-bin version/meme. cgi). The parameters are optimized as follows: zero or one, the rate of occurrence of a pattern on each sequence; $10 \mathrm{BP}$, module width range; and three other broad ranges of patterns were identified, and finally 10 motifs were selected. All different parameters follow with default values. In the same way, the distribution of the all conserved cis-elements of the promoter sequences of safflower $C t F 3^{\prime} H$ genes were extensively investigated by exploiting the PlantCARE software available at (https://sogo.dna.affrc.go.jp/).

\section{Transcriptomic profiling and qRT-PCR analysis}

The digital expression analysis of identified $C t F 3^{\prime} H$ genes in safflower was conducted using the RNA-seq metadata in four different flowering stages, root, seed, and stem tissues respectively. A combined heatmap was generated from the kilobase model of exon model per million mapped read (RPKM) values in each tissue under investigation. Furthermore, the expression level of each CtF3' $\mathrm{H}$ transcript was validated with qRT-PCR analysis using four different flowering stages of saflower. For this purpose, the primer pairs for these selected $22 \mathrm{CtF}^{\prime} \mathrm{H}$ genes were synthesized from the genomic data. The total RNA extraction was carried out from each flower tissue with the help of TRIzol reagent. The synthesis of cDNA templates were produced using the reverse transcriptase enzyme.

All qRT-PCR reactions were carried out with SYBR ${ }^{\circledR}$ Premix Ex Taq ${ }^{\text {TM }}$ (TliRNaseH Plus) from TaKaRa Biotechnology Co., Ltd. (Dalian, China) using a real-time PCR device (Biosystems 7500). Following the manufacturer's protocol, a $20 \mu \mathrm{L}$ of PCR mixture was prepared including $1.0 \mu \mathrm{L} c D N A, 0.4 \mu \mathrm{M}$ each primer (F/R), 0.4 ROX dye, $10 \mu \mathrm{l}$ main mixture, and RNase free water $7.8 \mu \mathrm{L}$. The internal reference gene $18 \mathrm{~s}$ ribosomal RNA of safflower was used as control. The relative expression level for each transcript was 
measured according to $2^{-\Delta \Delta C t}$ method (Min et al. 2020). The primer details are listed in (Supplementary Table S1).

\section{Gene cloning and subcellular localization of CtF3'H5}

The flower petals of the safflower were stored at $\mathrm{t}-80^{\circ} \mathrm{C}$ and then ground in liquid nitrogen and then subject to total RNA extraction with TRIzol reagent. RNA concentrations were determined by NanoDrop 2000 based on OD260 / 280 values and checked by electrophoresis on 1\% agarose gel. Following the manufecturer's instructions, reverse transcription was carried out to synthesize the first strand cDNA templates for PCR amplification. The gene specific primers (Supplementary Table S2) were designed according to the genomic sequence of $C t F 3^{\prime} H 5$. The PCR reaction conditions were as follow: $98^{\circ} \mathrm{C}$ for 10 seconds, $98^{\circ} \mathrm{C}$ for 10 seconds, $65^{\circ} \mathrm{C}$ for 15 seconds, $72^{\circ} \mathrm{C}$ for 2 minutes, 35 cycles; $72^{\circ} \mathrm{C}$ for 10 minutes. The amplified PCR products of $\mathrm{CtF} 3^{\prime} \mathrm{H} 5$ gene were detected on $1 \%$ agarose gel electrophoresis and the results were recovered using a gel recovery kit and then ligated to pEASY-T1 Simple vector, and then transformed into the competent cells of bacteria. The positive colonies of pEASY-T1-CtF3'H5 were selected on LB agar plates and the results was confirmed with half colony PCR method using gene specific primers. After PCR confirmation, the plasmids was extracted from the positive colonies and further confirmed with double restrictiomn enzyme digestion, and then sent to Shanghai Biotech Engineering Services Co., Ltd for sequencing. Similarly, using the CtF3'H5 T vector plasmid, the full length cDNA sequence of CtF3'H5 was amplified using a new primer pairs (Table S2) containg Bglll, and Ncol dual restriction sites, and the target band was subcloned into a new plant expression vector pCAMBIA1302-GFP-35S for the investigation of subcellular localization. The recombinant plasmid was confimred initially with double restriction digestion using Bglll and Ncol enzymes and then verified by sequencing. The recombinant plasmid (pCAMBIA1302-GFP-CtF3'H5) was then transformed into agrobacterium EHA105 competent cells using heat and shock method and the positive bacterial strains were selected on YEP agar medium. The transient transformation system was established using the agrobacterium mediated infection of the onion epidermal cell under controlled conditions. The Agrobacterium-infected onion epidermal cells containing the recombinant plasmid (pCAMBIA1302-GFPCtF3'H5), were plated on the solid medium of $1 / 2 \mathrm{MS}$, placed in dark culture medium at $28^{\circ} \mathrm{C}$ for 18 hours and then the results of the GFP expression was analyzed by scanning confocal laser microscopy.

\section{Prokaryotic expression and Western Blot}

The full-length cDNA sequence of CtF3'H5 was amplified using Pfu DNA polymerase Takara) with the help of a newly designed set of primers (Table S2) with an added Hind III and Xhol restriction sites. The complete fragment of the amplified $\mathrm{CtF} 3^{\prime} \mathrm{H} 5$ gene was cut and recovered, mixed with the prokaryotic expression vector $\mathrm{pET}-28 \mathrm{a}^{+}$in a 4:1 ratio, and then ligation was performed into the same digested empty pET28a + vector using T4 DNA ligase enzyme overnight at $16^{\circ} \mathrm{C}$ for $16 \mathrm{~h}$. The ligated product was transferred to the DH5a strain and recombinant plasmid cells were selected on kanamycin-resistant LB plate, and the positive clones were confirmed by PCR following double digestion sytem sequencing. The successfully constructed expression plasmid was named pET-28a-CtF3'H5, and the correctly sequenced plasmid was transformed into the competent BL21 cell. The positive clones were identified and cultured 
at a speed of $180 \mathrm{r} / \mathrm{min}$ at $37^{\circ} \mathrm{C}$ and $28^{\circ} \mathrm{C}$ respectively in $10 \mathrm{ml}$ of LB liquid medium containing $50 \mu \mathrm{g} / \mathrm{mL}$ of ampicillin. When the absorbance at $600 \mathrm{~nm}$ (A600) reaches $0.8,1 \mathrm{~mol} / \mathrm{L}$, the induction with IPTG was carried out using different concentration $(0.2,0.4,0.5,0.6,0.7,0.8)$ at different time periods including $2 \mathrm{~h}, 4$ h, 5 h, 6 h, and 8 h respectively. The soluble protein extract of CtF3'H5 was detected on 12\% SDS-PAGE, and then the target bands were stained with coomassie brilliant blue dye. The proteins obtained were further subjected to PVDF membrane for 2 hours, and the membrane was washed for 5 min using Tris buffer saline Tween (TBST) and then blocked with the blocking buffer (TBST buffer containing 5\% skim milk powder) for 1 hour. After performing the incubation with primary antibody at room temperature, we discarded the blocking buffer. The incubation was allowed at $4^{\circ} \mathrm{C}$ overnight followed by thorough washing with TBST 5 times for 5 min each time. The addition of the second antibody onto the membrane was enabled for 2 hours incubation following by washing 5 times with TBST for 5 min each time. The antibody binding complex was analyzed with the help of ECL chemiluminescence and this experiment was repeated three times.

\section{Statistical analysis}

All the experiments were conducted three times. Data were shown as the mean \pm standard deviation of three parallel experiments $(\mathrm{N}=3)$ with the standard deviation always less than $5 \%$.

\section{Results}

\section{Identification and Physicochemical Properties of the Safflower CtF3'H Genes}

To localize all putative $C t F 3^{\prime} H$ genes in safflower, we performed a series of electronic searches and Pfam ID scan for safflower genome database. The F3'H orthologes were also screened out from TAIR database for comparitive analysis. A total of 22 candidate $C t F 3^{\prime} H$ genes were identified in safflower geneome and was subjected to blast for validation. The physico-chemical properties of CtF3'H proteins were observed including the protein length ranging from 128aa to 518aa, the molecular weight ranges from $14.84 \mathrm{kDa}$ $\left(C t F 3^{\prime} H 22\right)$ to $58.6 \mathrm{kDa}\left(\mathrm{CtF3}^{\prime} \mathrm{H} 23\right)$, with the average is $40.75 \mathrm{kDa}$. The isoelectric point values ranged from 4.94 (CtF3'H7) to 9.49 (CtF3'H37) with an average of 7.14. Similarly, the analysis of grand average of hydropathicity (GRAVY) showed that the majority of CtF3'H proteins were hydrophilic, only CtF3'H37 with GRAVY 0.88 expressed hydrophilic. (Supplementary Table 1)

\section{Phylogenetic Analysis of CtF3'H family with Arabidopsis P450}

The protein-enconding CtF3'Hs with Arabidopsis AtP450s were assembled in a phylogenetic tree which is mainly divided into two main branches: type A and non-A. These two branches are clustered into nine subclasses including, clan71, clan75, clan81, clan76, clan78, clan72, clan97, clan77, clan51 and clan79 (Fig. 1). The detailed overview of phylogenetic analysis showed that clan71, clan72, clan77, clan79 and 
clan 81, clans51 are clans that do not contain the $C t F 3^{\prime} H$ gene family, whereas the other three clans unevenly contained members of $C t F 3^{\prime} H$ gene family. All putative $C t F 3^{\prime} H$ genes were identified as non- $\mathrm{A}$ type of Cytochrome P450s which is further classified into 22 subcategories. On the other hand, the subfamily CYP71 is the largest A-class (48.18\%) however, our analysis showed that it does not contain any genes of $C t F 3^{\prime} H$. The organization of clan 75 indicated the assembly of various other AtCYP450 grouped with 14 putative $C t F 3^{\prime} H$ genes. Similarly, clan 76 also showed a clear association between 8 AtCYP450 members and $10 \mathrm{CtF3} H$ genes of safflower. However, the distribution of genes at clan 78 showed 7 members of AtCYP450 grouped with 2 CtF3'H genes, but exceptionally, these 2 CtF3'H genes originated directly from the root of the tree located at the same branch as clan 78 revealing their ancestral divergence points.

\section{The distribution of conserved protein motifs and alignment of CtF3'H Proteins}

The organization and distribution of the conserved protein motifs of CtF3'H protein were extensively investigated and screened with MEME online tool version 5.1.1. The output results of the 22 putative CtF3' $H$ protein sequence were combined into a phylogenetic tree. We found two highly conserved protein motifs expressed as red and blue masses, correspondingly (Fig. 2). The location and distribution pattern also revealed that most of the conserved motifs were localized to $\mathrm{C}$ terminus of $\mathrm{CtF}{ }^{\prime} \mathrm{H}$ proteins. The subfamily members such as $\mathrm{CtF}{ }^{\prime} \mathrm{H} 9$ and $\mathrm{CtF} 3^{\prime} \mathrm{H} 37$ do not showed considerable conservation of the investigated protein motifs. Similarly, the composition of motifs conservation in $\mathrm{CtF}{ }^{\prime} \mathrm{H} 2 \mathrm{Z}$ described incomplete pattern. Notably, in most cases, the putative proteins of safflower $C t F 3^{\prime} H$ demonstrated the two conserved regions which usually linked next to each other; but, in the case of $C t F 3^{\prime} H 26$, these two conserved regions were found separated from each other. On the contrary side, some proteins indicated inadequate conservation modules such as $\mathrm{CtF3}^{\prime} \mathrm{H} 24$ and $\mathrm{CtF}^{\prime} \mathrm{H} 22$.

In addition, we also performed multiple sequence alignment of all putative $22 \mathrm{CtF}{ }^{\prime} \mathrm{H}$ proteins to determine their sequence homology (Fig. 3). The highly conserved homology within the same subfamily members was shown with red and blue color. The similarity of amino acid conservation pattern confirms the previous results of MEME scores obtained from CtF3'H proteins. The corresponding locations of each CtF3'H protein pattern were found conserved, however, motif 1 and motif 2 were found scattered among all $\mathrm{CtF} 3^{\prime} \mathrm{H}$ proteins. Meanwhile, the occurrence of motif 3 is present in all subtypes except $C t F 3^{\prime} H 1$ and CtF3'H2. Similarly, motif 4 is not present in $C t F 3^{\prime} H 7$ and part of $\mathrm{CtF}^{\prime} \mathrm{H} 8$ whereas motif 5 was found in some proteins such as $C t F 3^{\prime} H 1,3,4,5$, and 10 . The presence of motif 6 is found in $C t F 3^{\prime} H 1,3,4,6$ while motif 8 was only screened for the proteins of $C t F 3^{\prime} H 8,9$ and 10. Altogether, the distribution and conservation of signature protein motifs as well as the progressive alignment network revealed significant evolutionary and sequential features of CtF3'H subfamily in safflower.

\section{Organization of cis-regulatory units of CtF3'H promoters}

To identify different variants of gene promoter cis-regulatory elements, we extensively analyzed the $2 \mathrm{~kb}$ upstream to the ATG starting site of CtF3'H genes using the PlantCARE's software. The abundently 
annotated cis-elements within the promoter sequence of most of the $C t F 3^{\prime} H$ genes contained factors related to light reactions for example, 19/22 genes CtF3'H contain the G-Box cis-element, suggesting that signals induction might play crucial roles during the transcription activation and regulation of $C t F 3^{\prime} H$ genes in safflower (Fig. 4). Moreover, our analysis revealed the presence of MYB and MYC responsive elements which are actively implicated during flavonoid biosynthesis. In addition, the occurrence of cisregulatory units conserved in other major plant reactions have also been studied within the promoters of different $C t F 3^{\prime} H$ genes, such as drought (MBS), and abscisic acid reaction (ABRE). Convincingly, the presence of such types of cis-acting elements in safflower $C t F 3^{\prime} H$ genes suggested positive hallmarks during plant secondary metabolism and various stress related and hormonal induced pathways.

\section{Expression analysis of CtF3'H subfamily genes}

We conducted a meta-analysis of the data sets of 22 CtF3'H genes showing that the digital expression of all putative $C t F 3^{\prime} H$ genes exhibit a differential expression pattern through different developmental stages of safflower (Fig. 5A). The developmental stages include both the vegetative stages as well as the reproductive stages. Some $C t F 3^{\prime} H$ genes $\left(C t F 3^{\prime} H 5,20,32\right)$ seem to be expressed at all stages with strong expression specified in shoot tissues. However, some of the CtF3'H genes including (CtF3'H27, 1, 2, 13) were abundantly expressed in the root tissue/organs and also through different growth stages of the seeds. Furthermore, the expression level of $C^{2} F 3^{\prime} H 33,20,37,28,34$ showed highest expression in the intermediate seed maturation stage, besides $\left(C t F 3^{\prime} H 23,24,33\right)$. Specifically, the abundance of $C t F 3^{\prime} H 22$ appears only in the seeds development stages. In addition, to confirm the expression level of these putative $C t F 3^{\prime} H$ genes and their correlation the during flower petals development experimentally, we conducted the qRT-PCR analysis of 22 putative $C t F 3^{\prime} H$ genes previosly obtained from safflower genome using four different flowering stages (Fig. 5B). The highest expression level was observed for CtF3'H23 at the fading stage of flower development. The relative expression level of $C t F 3^{\prime} H 5$ was uniformly detected during all four stages of flowering. The transcript abundance of almost all putative $C t F 3^{\prime} H$ genes demonstrated a differential pattern of transcription with slight differences e.g. highest expression in the flowering period, and descending in the initial, bud, and fading stages. Our qRT-PCR results showed that the fold change values of the CtF3'H genes were in accordance with the prediction of the digital expression presented earlier. The expression assay of $C t F 3^{\prime} H$ genes during flower development in safflower suggested a variable transcription regulation pattern highlighting their decisive roles during petal development in safflower.

\section{Cloning and Subcellular Localization of CtF3'H5}

The coding region of $C t F 3^{\prime} H 5$ target gene encoding 455 amino acids was cloned from safflower using Taq polymerase enzyme and the result was confirmed on $1 \%$ agarose gel electrophoresis and sanger sequencing. The sequencing results show that the obtained sequence is consistent with the expected result of the $\mathrm{CtF} 3^{\prime} \mathrm{H} 5$ gene, indicating that the gene has been successfully cloned. (Fig. 6A). The protein sequence of $C t F 3^{\prime} H 5$ was subjected to multiple amino acid sequence alignments with various other F3' $\mathrm{H}$ proteins obtained from 14 different species including callistephus chinensis, camellia sinensis, dahlia pinnata and centaurea cyanus etc (Fig. 6B). It was found that the amino acid sequence of CtF3'H5 
contained the common conserved "GGEK" sequence at positions 434 to 437, which is the specific to F3'H gene. The next conserved sequence "LPPGP" starting at position 39 which is the main conserved domain of cytochrome P450 was also found confirming the authenticity of P450 system. The 3D structure prediction of CtF3'H5 target protein was performed using the SWISS-MODEL online software (Fig. 6C) (Lu et al. 2018) by translating the genomic sequence of CtF3'H5 into amino with the help of Expasy ProtParam 4.0 online tool. The theoretical molecular weight was observed as $50.03 \mathrm{kD}$ with the average total hydrophobic value $-0,035$ suggesting that $C t F 3^{\prime} H 5$ is a hydrophilic protein.

In addition, the subcellular localization prediction for $C t F 3^{\prime} H 5$ was carried out by exploiting the online webserver of ProtComp version 9.0, indicated the predominant signals to the plasma membrane. However, to confirm the experimental status of the subcellular localization of our target CtF3'H5 protein, we therefore, investigated the transient expression system using the fusion vector of pCAMBIA1302 and CtF3'H5 constructing pCAMBIA1302-CtF3'H5-GFP vector. The aforesaid recombinat vector was transformed into onion epidermal cells by agrobacterium mediated infection system under controlled conditions. Our findings marginally demonstrated that the GFP signals were largely appeared within the plasma membrane section as compare to other parts of the cell (Figs. 7D-F). However, on the other hand, the GFP signals in the control vector indicated a dispersed pattern throughout the cell (Fig. 7A-C).

\section{Prokaryotic expression and Western Blot analysis of CtF3'H5}

The quantitative realtime expression analysis of $\mathrm{CtF3} H 5$ was investigated in different flowering stages of safflower as shown in (Fig. 8A). The results showed that $C t F 3^{\prime} H 5$ exhibits highest expression at the flowering stage of safflower followed by the initial stage. However, the remaining stages of flowering demonstrated lower expression level. These findings suggested the correlation of $C t F 3^{\prime} H 5$ with red and yellow color connotation in the safflower petals which is morphologically appeared during the flowering and initial stages. In the next phase, we cloned the full length $C t F 3^{\prime} H 5$ gene and ligated the final product into the expression cassttes of pET28a+ prokaryotic expression vector. The construction of the recombinant vector ( $\mathrm{pET} 28 \mathrm{a}^{+}-\mathrm{CtF} 3^{\prime} \mathrm{H5}$ ) was confiormed with double restriction digestion system (Fig. 8B\&C) and further accuracy was confirmed with sequencing. In the follow up experiments, the heterologously expressed CtF3'H5 protein was induced by treating the recombinant bacterial cells of BL21 strain carrying the (pET28a $\left.{ }^{+}-C t F 3^{\prime} H 5\right)$ vector with different concentrations of IPTG for different time periods. After the induction, the target protein product was confirmed on coomassie blue staining SDSPAGE which resulted in the expected band size of $50.3 \mathrm{Kda}$ however, it was observed that that the use of different IPTG concentrations did not influence the expression level of the protein in bacterial system (Fig. 8D). Similarly, the denaturation of the herologously expressed CtF3'H5 protein at different IPTG concentrations was performed using western blot hybridization method. As described (Fig. 8E), we observed a unique band of the same size ( $50.3 \mathrm{Kda})$, however, a variable expression level was found at different IPTG concentration. These results stringly suggested that the target CtF3'H5 protein is stably expressed in the bacterial system but its expression level could be marginaly influenced when induced with different concentrations of IPTG. 


\section{Discussion}

The flavonoid metabolism is one of the important secondary metabolic pathways in higher plants(Dermauw et al. 2020). In plants, F3'H plays an important role in determining flower color, seed pods and the color of stems and leaves. The ratio of F3' $H$ to F3'5'H determines the color level of grapes ( $D$ and $D$ 2011). In tea tree, catechin, a metabolite in the flavonoid pathway, may help the body improve its ability to fight cancer, antioxidants, mutants, tumors and cardiovascular disease. Flavonoid 3'hydroxylase is a key enzyme in the flavonoid metabolism pathway, which can catalyze the hydroxylation of position 3 of the B-ring of naringenin and dihydro kaempferol. Naringenin and dihydro kaempferol are oxidized to form a series of important intermediates in the flavonoid pathway. The structural stability and antioxidant function of these intermediates are closely related to the F3'H enzyme (Wei et al. 2015) identified naringenin as the optimal substrate for CsF3'H in enzyme activity experiments(Lv et al. 2017; Wang et al. 2014). In this study, we presented a breif overview of the genome wide identification and several structural and functional characteristics of CtF3'H subgene family in safflower. We have combined 110 Arabidopsis P450 genes and 22 identified $C t F 3^{\prime} H$ genes from the safflower genome to study their phylogenetic relationship resulted in two main classes A-type and non-A type (Fig. 1). Further classifications were grouped into nine clans of the phylogenetic tree, which was found consistent with the previous studies of the plant P450 family (Barvkar et al. 2012). Based on the evolutionary pattern, the CtF3'H5 gene family in safflower was recognized in the CYP clan 75,76.78 respectively. In previous studies on the F' ${ }^{\prime} \mathrm{H}$ monocot plants, they were also clearly classified with two separate states. CYP78 is one of the P450 families involved in flavonoid biosynthesis.

Flavonoid biosynthesis are tightly regulated by different spatial and time signals that can limit the accumulation of these compounds in plants (Musiol-Kroll et al. 2019). A number of studies have demonstrated the role of $\mathrm{F}^{\prime} \mathrm{H}$ gene in regulating plant metabolism and changes in flavonoid composition in the color of fower petals(Ueyama et al. 2002). Hence, the transcription regulation of F3'H gene may lead to signifiicant amount of understanding of the flavonoid pathway in plants. For this purpose, we extensively conducted the expression analysis of all identified $C t F 3^{\prime} H$ genes identifed in the safflower genome at different flowering stage (Fig. 5). Our findings confirmed significant differences during the transcription of $C t F 3^{\prime} H$ genes at different flowering stages in safflower reaching to its peak at flowering stage. Initially when the expression pattern was downregulated at bud stages where the pigment content is lower. However, the assumption was established on the basis of initial flowering and flowering stages, which is highest because the color of the petals appeared as red and yellow. Nontheless, the expression level of few $C t F 3^{\prime} H$ genes was not significant and quite faint including $C t F 3^{\prime} H 1, C t F 3^{\prime} H 2$ suggesting the synergetic effect of $C t F 3^{\prime} H$ mutation which limit some steps in favonoid biosynthesis ( $Y$ et al. 2019). The results of the $C t F 3^{\prime} H 5$ transcript showed a uniformly expression scheme in all four stages and the highest in the flowering period, this result was also found consistent with the digital expression prediction in the heatmap (Fig. 5).

We further characterized a putative $C t F 3^{\prime} H 5$ by cloning the full length cDNA sequence of this gene and performed multiple functional analyses to confirm its integrity with respect to flower pigmentation in 
safflower (Fig. 6). A variety of conserved amino acid sequences suggested that $C t F 3^{\prime} H 5$ is highly conserved as in other plant species ( $M$ et al. 2014). The subcellular localization study is probably one of the essential links to estimate the final function of a target protein (Naqvi et al. 2016). Hence, we experimentally investigated the localization of the $\mathrm{CtF}{ }^{\prime} \mathrm{H} 5$ target protein using the onion epidermal cells, through Agrobacterium mediated transformation by transiently expressing the fusion construct of pCAMBIA1302-CtF3'H5 containing the GFP cassettes (Fig. 7). The result suggested that the target protein of $C t F 3^{\prime} H 5$ was detected in the plasma membrane of the onion cell epithelial cells, disagreeing with the previous result of (Chen et al. 2017), who reported the F3'H gene on Brassica napus was localized into endoplasmic reticulum. This differences may occur due to the chromosomal position of the gene which is varies in saflower as compare to Brassica napus, however, the function of this protein was generally ascribed to flavonoid biosynthesis.

The enzymatic activity of Cytochrome P450 system exhibits a unique oxidative and reductive pattern. Nevertheless, to comprehend the vast substrate specificity of complex P450 proteins, the establishment of stable and cost effective expression system is yet a challenging task. The benefit of bacterial P450 expression systems has been covered by (Zelasko et al. 2013) presenting a fast and accurate expression system. In this study, we demonstrated a competent and stable expression system to produce a heterologously expressed CtF3'H5 protein by exploiting the prokaryotic expression system of bacteria (Fig. 8D). Our results identified an expected target protein of $C t F 3^{\prime} H 5$ expressin by a competent strain of bacteria (BI21) comprising a $50.3 \mathrm{kDa}$ size of band size in the SDS PAGE analysis. The level of CtF3'H5 target protein induction with IPTG indicated a stable production of the target protein however, with the use of different IPTG concentrations could possibly affect the expression of our target protein (Wei and Chen 2018), as described in our western blot hybridization analysis (Fig. 8E). Our findings from these analyses provide the foundation for screening highly expressive putative F3' $\mathrm{H}$ genes and identifying their related functions in plants which will be helpful to explain the underline rationale of this exceptionally important class of gene family.

\section{Declarations}

\section{Author's contributions}

Experimental work and data analysis was conducted by $\mathrm{NH}, \mathrm{KJ}$ and NA. Software and formal analysis were carried out by $Y Z, M X$ and ZX. Statistical analysis was performed by LX,WN, LJ and JL. The project was designed and supervised by LX, YN. The manuscript draft was prepared by NA. All authors read and approved the final manuscript.

\section{Acknowledgments}

This work was funded by grants from the National Natural Science Foundation of China (No. 31771868, 31501366), the Science and Technology Development Project of Jilin Province (No. 20190201172JC, 20190201175JC, 20150623024TC-11), Student innovation and entrepreneurship training program of Jilin Agricultural University. 


\section{Conflicts of Interest}

The authors declare no conflict of interest

\section{References}

Alaiye A, Kaya E, Pnarbal MZ, Harmanc N \& Cingi C (2020) An Experimental Comparison of the Analgesic and Anti-Inflammatory Effects of Safflower Oil, Benzydamine $\mathrm{HCl}$, and Naproxen Sodium. Journal of Medicinal Food 23(8)

Ambreen H, Kumar S, Kumar A, Agarwal M, Jagannath A \& Goel S (2018) Carthamus tinctoriusAssociation Mapping for Important Agronomic Traits in Safflower ( L.) Core Collection Using Microsatellite Markers. Frontiers in plant science 9:402 doi:10.3389/fpls.2018.00402

Barvkar VT, Pardeshi VC, Kale SM, Kadoo NY \& Gupta VS (2012) Phylogenomic analysis of UDP glycosyltransferase 1 multigene family in Linum usitatissimum identified genes with varied expression patterns. Bmc Genomics 13(1):175-175

Cerqueira A, Santana I, Araújo J, Lima H, Batatinha M, Branco A, Santos Junior M \& Botura M (2021) in vitroHomology modeling, docking, molecular dynamics and studies to identify acetylcholinesterase inhibitors. Journal of biomolecular structure \& dynamics:1-11 doi:10.1080/07391102.2021.1889666

Chen K, Liu H, Lou Q \& Liu Y (2017) Muscari armeniacumEctopic Expression of the Grape Hyacinth () R2R3-MYB Transcription Factor Gene, , Induces Anthocyanin Accumulation in Tobacco. Frontiers in plant science 8:965 doi:10.3389/fpls.2017.00965

Cho H, Kim O, Lee Y, Kang L, Nguyen C, Ishihara A \& Kim H (2017) Feruloylserotonin inhibits hydrogen peroxide-induced melanogenesis and apoptosis in B16F10 and SK-Mel-2 melanoma cells. Biochemical and biophysical research communications 491(4):973-979 doi:10.1016/j.bbrc.2017.07.158

Choudhary D, Pandey A, Adhikary S, Ahmad N \& Trivedi R (2016) Genetically engineered flavonol enriched tomato fruit modulates chondrogenesis to increase bone length in growing animals. Scientific Reports $6: 21668$

D N \& D W-R (2011) A P450-centric view of plant evolution. The Plant journal : for cell and molecular biology 66(1):194-211 doi:10.1111/j.1365-313X.2011.04529.x

De Palma M, Fratianni F, Nazzaro F \& Tucci M (2014) Isolation and functional characterization of a novel gene coding for flavonoid 3'-hydroxylase from globe artichoke. Biologia Plantarum 58(3):445-455

Dermauw W, Van Leeuwen T \& Feyereisen R (2020) Diversity and evolution of the P450 family in arthropods. Insect biochemistry and molecular biology 127:103490 doi:10.1016/j.ibmb.2020.103490 
Du C, Hou J, Wang C, Zhang M, Zheng Y, Yang G \& Hu Y (2021) Effects of safflower yellow on cholesterol levels in serum and brain tissue of APP/PS1 mice. Metabolic brain disease 36(4):557-569 doi:10.1007/s11011-021-00680-0

Gao S, Xu X, Zeng W, Xu S, Lyv Y, Feng Y, Kai G, Zhou J \& Chen J (2020) SEfficient Biosynthesis of (2)Eriodictyol from (2)-Naringenin in through a Combination of Promoter Adjustment and Directed Evolution. ACS synthetic biology 9(12):3288-3297 doi:10.1021/acssynbio.0c00346

Guo L, Gao L, Ma X, Guo F, Ruan H, Bao Y, Xia T \& Wang Y (2019) Functional analysis of flavonoid 3'hydroxylase and flavonoid 3',5'-hydroxylases from tea plant (Camellia sinensis), involved in the B-ring hydroxylation of flavonoids. Gene 717:144046 doi:10.1016/j.gene.2019.144046

Lin K, Qin Z, Qu C, Chen X, Jiang Q, Li M, Zheng Q \& Li D (2021) Hydroxyl safflower yellow B combined with doxorubicin inhibits the proliferation of human breast cancer MCF-7 cells. Oncology letters 21(5):426 doi:10.3892/ol.2021.12687

Lu R, Yuan T, Wang Y, Zhang T, Yuan Y, Wu D, Zhou M, He Z, Lu Y, Chen Y, Fan J, Liang J \& Cheng Y (2018) Spontaneous severe hypercholesterolemia and atherosclerosis lesions in rabbits with deficiency of lowdensity lipoprotein receptor (LDLR) on exon 7. EBioMedicine 36:29-38 doi:10.1016/j.ebiom.2018.09.020

Lv GY, Guo XG, Xie LP, Xie CG, Zhang XH, Yuan Y, Lei X, Tang YY, Pan XL \& Guo AG (2017) Molecular Characterization, Gene Evolution, and Expression Analysis of the Fructose-1, 6-bisphosphate Aldolase (FBA) Gene Family in Wheat (Triticum aestivum L.). Frontiers in Plant Science 8

M B, S B, A W, K A, G S, T S, F K, T GC, M B, L B \& T S (2014) SWISS-MODEL: modelling protein tertiary and quaternary structure using evolutionary information. Nucleic acids research 42:W252-8 doi:10.1093/nar/gku340

Min F, Sun H, Wang B, Ahmad N \& Li H (2020) Hepatoprotective effects of hydroxysafflor yellow A in Dgalactose-treated aging mice. European Journal of Pharmacology:173214

Musiol-Kroll EM, Tocchetti A, Sosio M \& Stegmann E (2019) Challenges and advances in genetic manipulation of filamentous actinomycetes - the remarkable producers of specialized metabolites. Natural Product Reports 36(12)

Nagamatsu A, Masuta C, Matsuura H, Kitamura K, Abe J \& Kanazawa A (2009) Down-regulation of flavonoid 3'-hydroxylase gene expression by virus-induced gene silencing in soybean reveals the presence of a threshold mRNA level associated with pigmentation in pubescence. Journal of Plant Physiology 166(1):0-39

Naqvi AAT, Anjum F, Khan FI, Islam A \& Hassan MI (2016) Sequence Analysis of Hypothetical Proteins from Helicobacter pylori 26695 to Identify Potential Virulence Factors. Genomics Informatics 14(3)

Panche, Diwan \& Chandra (2016) Flavonoids: an overview. Journal of Nutritional Science 
Ren T, Zheng P, Zhang K, Liao J, Xiong F, Shen Q, Ma Y, Fang W \& Zhu X (2021) Effects of GABA on the polyphenol accumulation and antioxidant activities in tea plants (Camellia sinensis L.) under heat-stress conditions. Plant physiology and biochemistry : PPB 159:363-371 doi:10.1016/j.plaphy.2021.01.003

Schrago C, Aguiar B \& Mello B (2018) Comparative evaluation of maximum parsimony and Bayesian phylogenetic reconstruction using empirical morphological data. Journal of evolutionary biology 31(10):1477-1484 doi:10.1111/jeb.13344

Ueyama Y, Suzuki Kl, Fukuchi-Mizutani M, Fukui Y \& Tanaka Y (2002) Molecular and biochemical characterization of torenia flavonoid 3'-hydroxylase and flavone synthase II and modification of flower color by modulating the expression of these genes. Plant Science 163(2):253-263

Wang X, Xu Y, Chu C, Li H, Mi J, Wen Z, Zhang S, Wang Q \& Quan S (2019) Effect of safflower yellow on early type II diabetic nephropathy: a systematic review and meta-analysis of randomized controlled trials. Journal of Pediatric Endocrinology \& Metabolism Jpem 32(7):653

Wang Y-S, Xu Y-J, Gao L-P, Yu O, Wang X-Z, He X-J, Jiang X-L, Liu Y-J \& Xia T (2014) Functional analysis of Flavonoid 3',5'-hydroxylase from Tea plant (Camellia sinensis): critical role in the accumulation of catechins. Bmc Plant Biology 14(1):347

Wei K \& Chen H (2018) Global identification, structural analysis and expression characterization of cytochrome P450 monooxygenase superfamily in rice. BMC genomics 19(1):35

Wei K, Wang L, Zhang C, Wu L, Li H, Zhang F \& Cheng H (2015) Transcriptome Analysis Reveals Key Flavonoid 3'-Hydroxylase and Flavonoid 3',5'-Hydroxylase Genes in Affecting the Ratio of Dihydroxylated to Trihydroxylated Catechins in Camellia sinensis. PloS one 10(9):e0137925 doi:10.1371/journal.pone.0137925

Y H, S V, RE S-G, S R-M, D Z, AV L \& SS K (2010) Ectopic expression of apple F3'H genes contributes to anthocyanin accumulation in the Arabidopsis tt7 mutant grown under nitrogen stress. Plant physiology 153(2):806-20 doi:10.1104/pp.109.152801

Y J, B L, Y Z, X Z, Y X \& C L (2019) Evolutionary dynamic analyses on monocot flavonoid 3'-hydroxylase gene family reveal evidence of plant-environment interaction. BMC plant biology 19(1):347 doi:10.1186/s12870-019-1947-z

Zelasko S, Palaria A \& Das A (2013) Optimizations to achieve high-level expression of cytochrome P450 proteins using Escherichia coli expression systems. Protein expression and purification 92(1):77-87

\section{Figures}




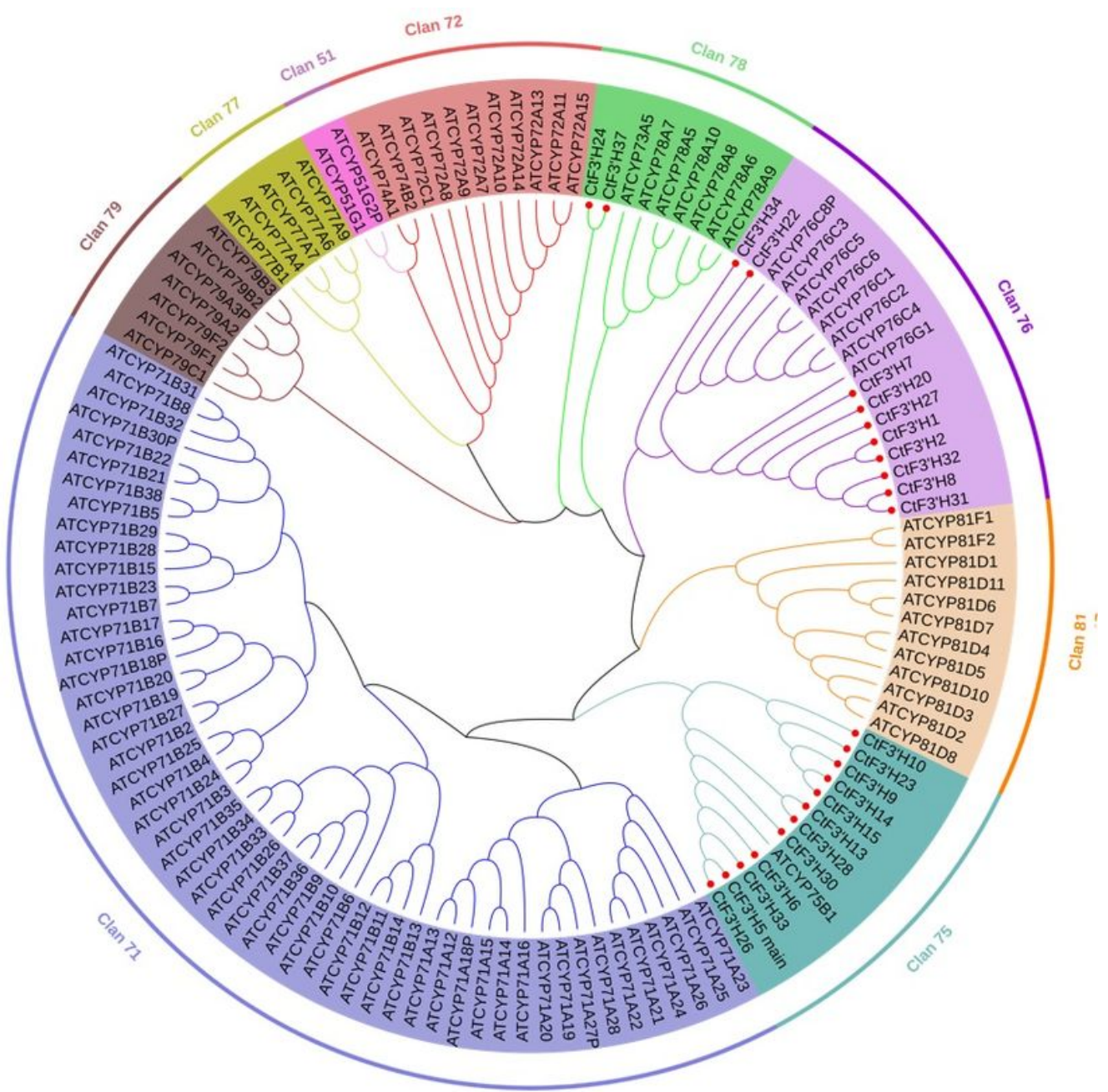

\section{Figure 1}

Phylogenetic Analysis of 22 CtF3'H and 110 CYP450 from Arabidopsis Thaliana Phylogenetic tree is constructed by using MEGA-X with 1000 bootstrap replicates: Different lineages are represented by different colors. The abbreviations used for different plant protein chains are as follows: At - Arabidopsis thaliana) (Ct - Carthamus tinctorius) 

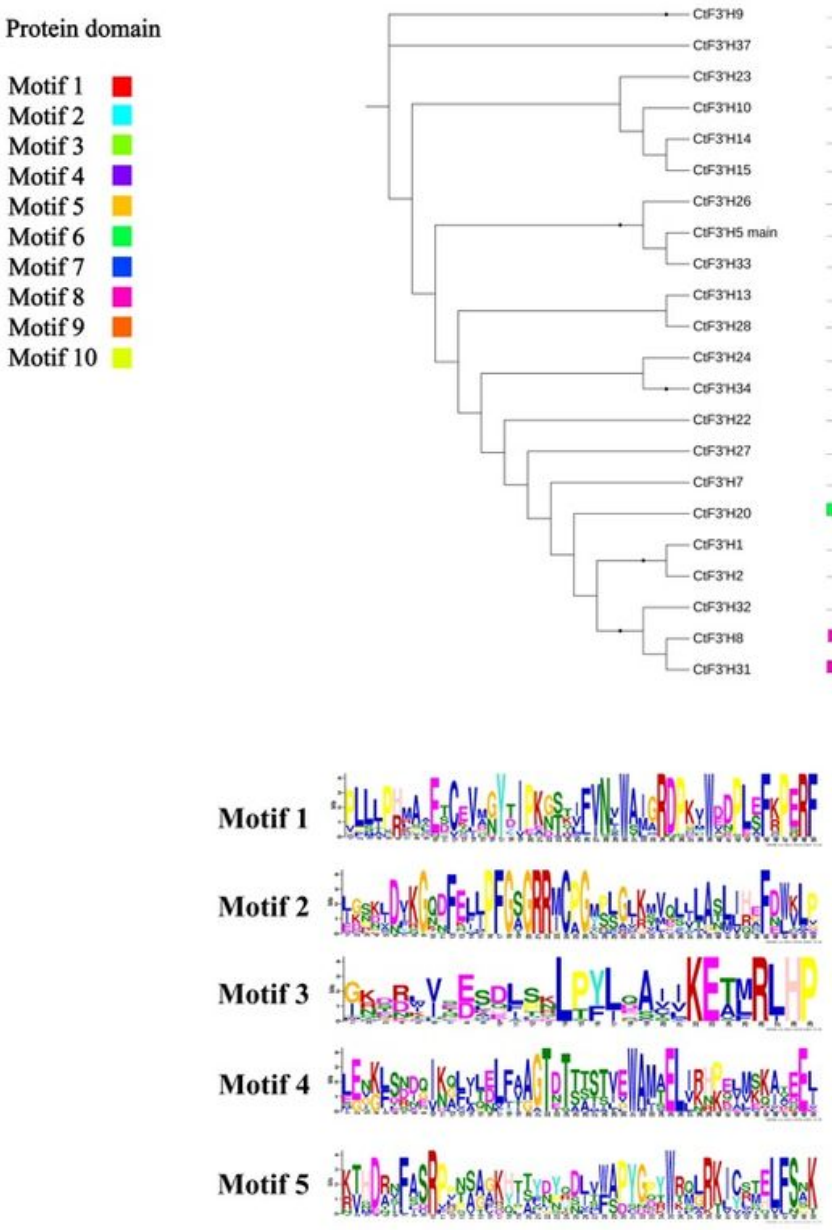
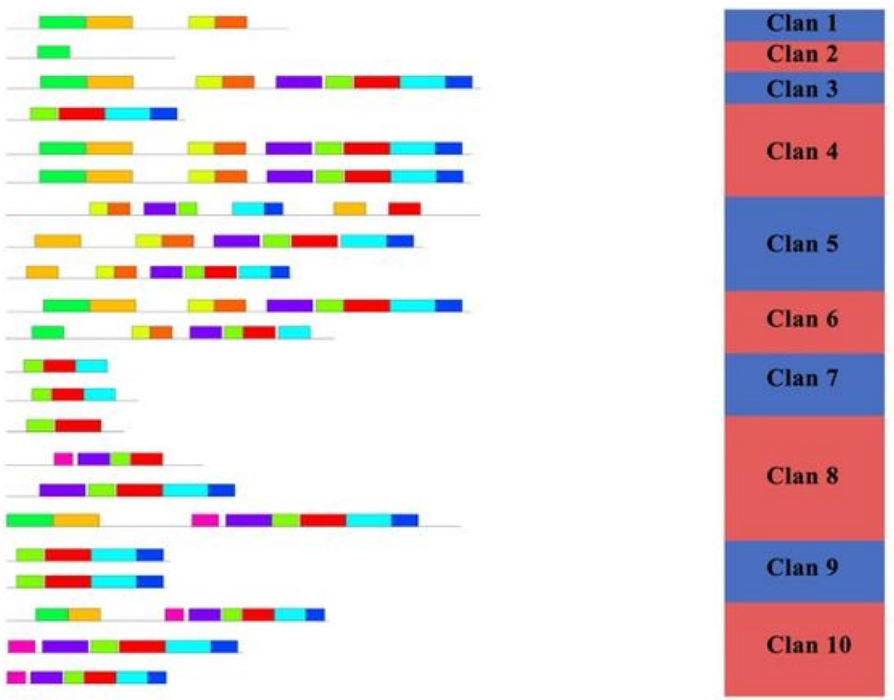

\section{Figure 2}

The conserved protein motifs in CtF3'H protein obtained from safflower genome. The online webserver of MEME was exploited to identify the conserved patterns of CtF3'H genes. 
CtF3'H1 NLWAMGRDPKVWDDPLSFKPERFIG . . . . SKVDF KGQDFELLPFGSGRRMCPGMP: CtF3' H2 NLWAMGRDPKVWDDP LSFKPERFIG . . . . SKVDF KGQDFELLPF GSGRRMCPGMP: CtF3' H5 NVWAI ARDPKMWVDP LEERP SRFLPG. GEK PNSNVKGNDE EI IPF GAGRR I AAGMS: CtF3' H7 NVWAI GRDPKVWEDP L SEKPERELG . . . L LDLDF KGHDF EFIPF GAGRRMCPGVP! CtF3' H8 NLWAMGRDPKVWDDP LSEKPERFVD . . . . SKLDI KGQDEELLPF GSGRRMCPGMP: CtF3'H9 CtF3'H10 CtF3'H13 CtF3'H14 CtF3'H15 CtF3' H2O

NVWTSIMRDSDYW̄DDPKAFRPERFLG . . . . KDIDVKGKHFELIPFGSGRRMCPGYM: NVWAL GRDPNHWENPLEFRPERFEK . . . . . NQLDVRGQHF HMLPF GSGRRMCPGT S: NSWSL GRDPGVWVDS EAFRPERFLG . . . . . RDIDVKGQHFELLPF GSGRRMCPGYS: NSWSI GRDPGVWVDPEVERPERFLG . . . . . RDIDVKGQHFELLPF GSGRRMCPGYS: NIWAI GRDPK IWNDP LSEKP ERFLG...... SKIDFRGQNE EFIPFGSGRRMCPGL P.

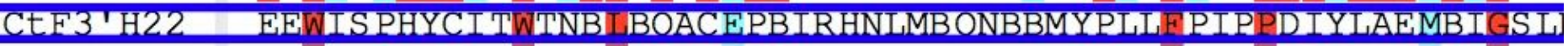
CtF3' H23 STWTLGRDPQLWDNPLEFCPERFIE . . . . . NEIDMKGTNF KFMPF GAGRRMCPGYS: CtF3 ' H24 NVWAI YRDPKNWPDP LEFKPERFLN . . . . NKWDYNGKNLKFLPF GVGRR ICPGI P: CtF3' $\mathrm{H} 26$ CtF3' $\mathrm{H} 27$ CtF3'H28 CtF3' H31 CtF3'H32 CtF3'H33 CtF3'H34 CtF3'H37 NVWAI ARDPKMWANP LEFQP NRFLPG. GEK PNANV KGNDFEI IPF GAGRR ICAGMS: NLWAMGRDPK IWDDP LSFKPERFIG . . . . SKLDY KGQDFELFAI WFRKE DVSRL T: NIWS I GRNPKYWESP LEFKPRRF IE GDALKSPLDI KGQSFQLLPFGTGRRGCPGIN: NLWAMGRDPKVWDDP LSEKPERFIG . . . . . SKLDI KGQDE ELLPF GSGRRMCPGMP: NIWAMARDPKVWDDP LSFKPERFID . . . . . SKLDI KGQDFELLPF GSGRR ICPGMP: NVWAI ARDPKMWANP LEFQPNRELPG. GEK PNANV KGNDE EIIPF GAGRR ICAGMS: NAWAI HRDPE IWEKP LEEWPERFID . . . . NKLDY SGNDF NYFPF GSGRR ICAGQRI

\section{Figure 3}

The analysis of multiple sequence alignment of CtF3'H proteins in safflower . The color boxes indicate 70 and $50 \%$ of the amino acid identity. 
I RY-eleent

I MBS

I G-Box

I ABRE

I MYC

MYB
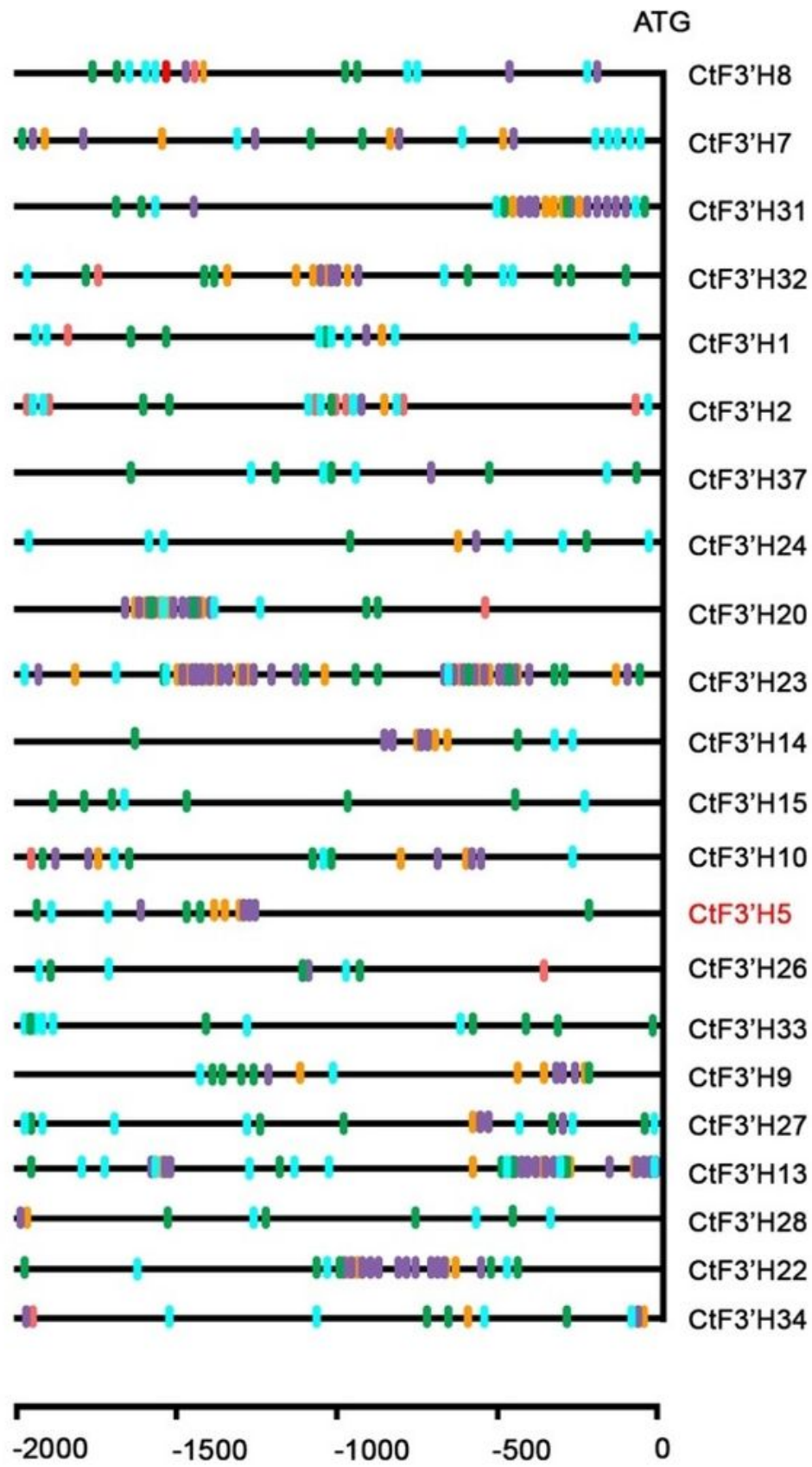

Figure 4

The graphical illustrations of key cis-regulatory units found in the promoter region of CtF3'H genes from safflower. 


\section{(A)}

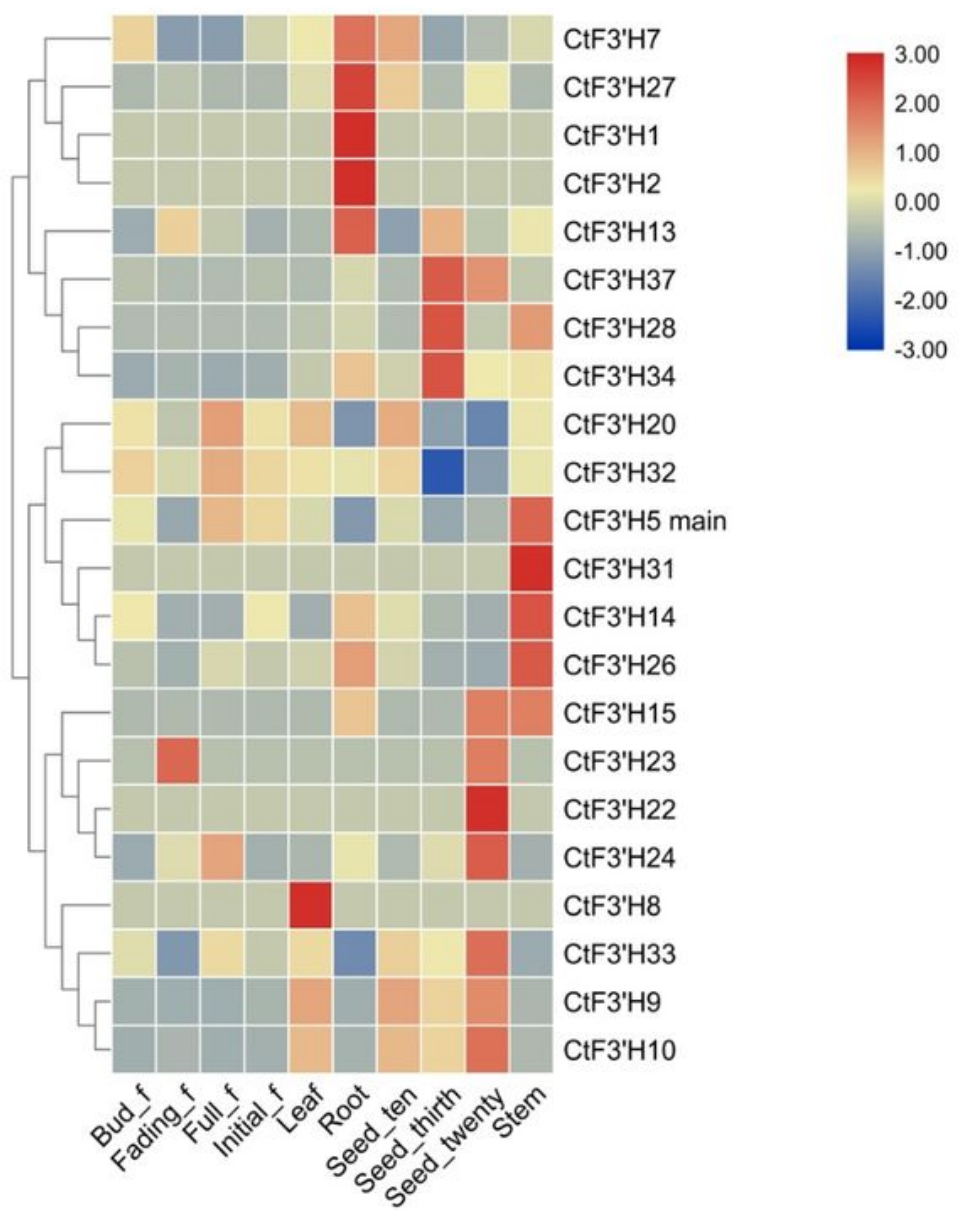

(B)

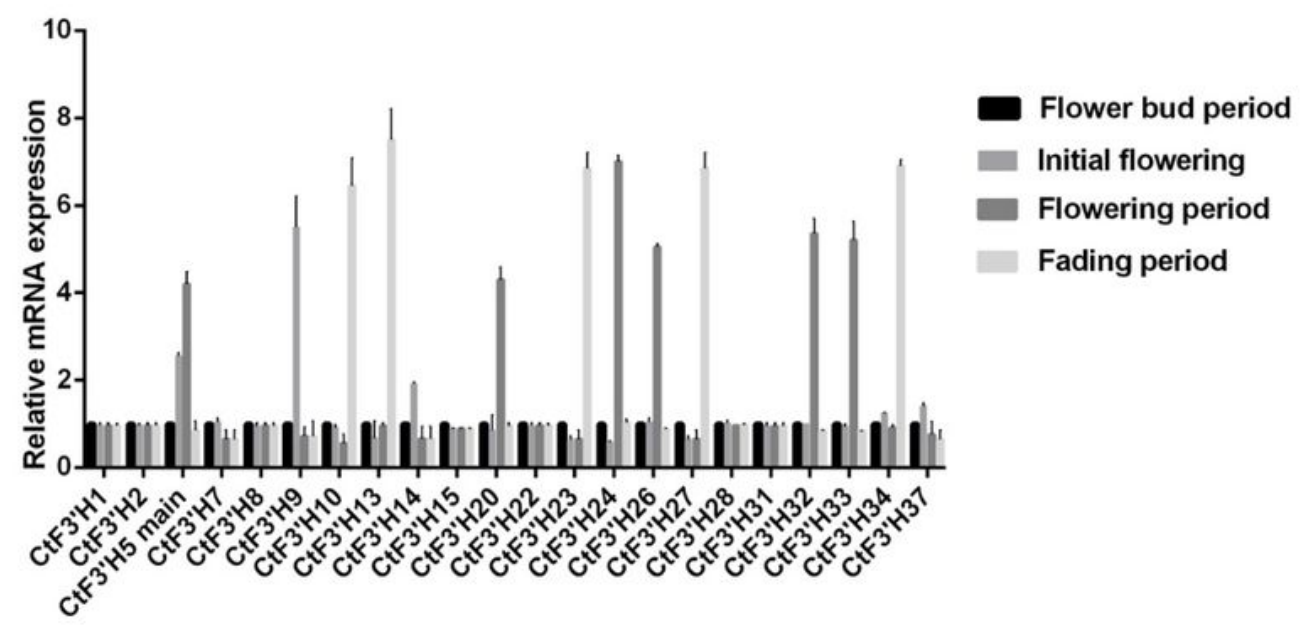

Figure 5

(A) Heat map representing the digital expression profile CtF3'H genes. The map shows the development time and expression of CtF3'H in each development stage of Safflower. (B) Expression profiles of 22 putative CtF3'H genes in different flowering stages of safflower were determined by qRT-PCR analysis. Different background colors represent different periods of the safflower CtF3'H gene. Expression analysis 
was standardized using the $18 \mathrm{~S}$ rRNA gene as an internal reference. Error bars are estimated from differences in the expression patterns of three independent biological samples.

(A)
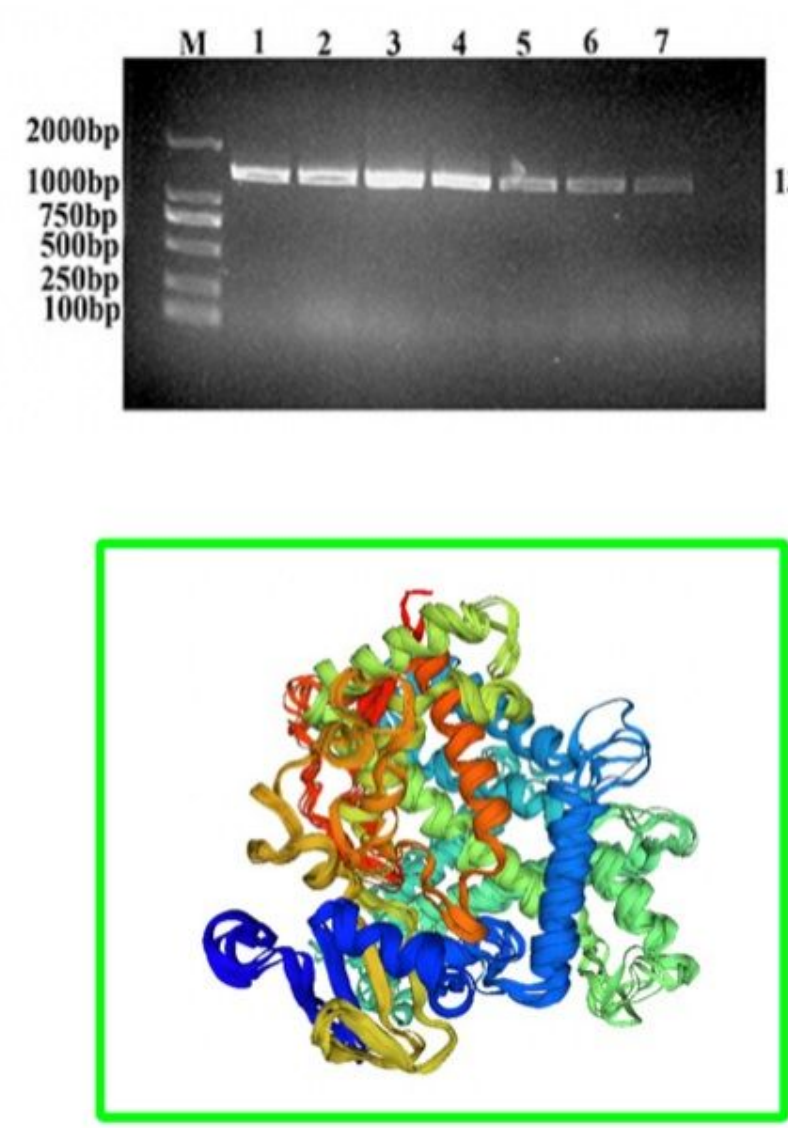

(C)
(B)
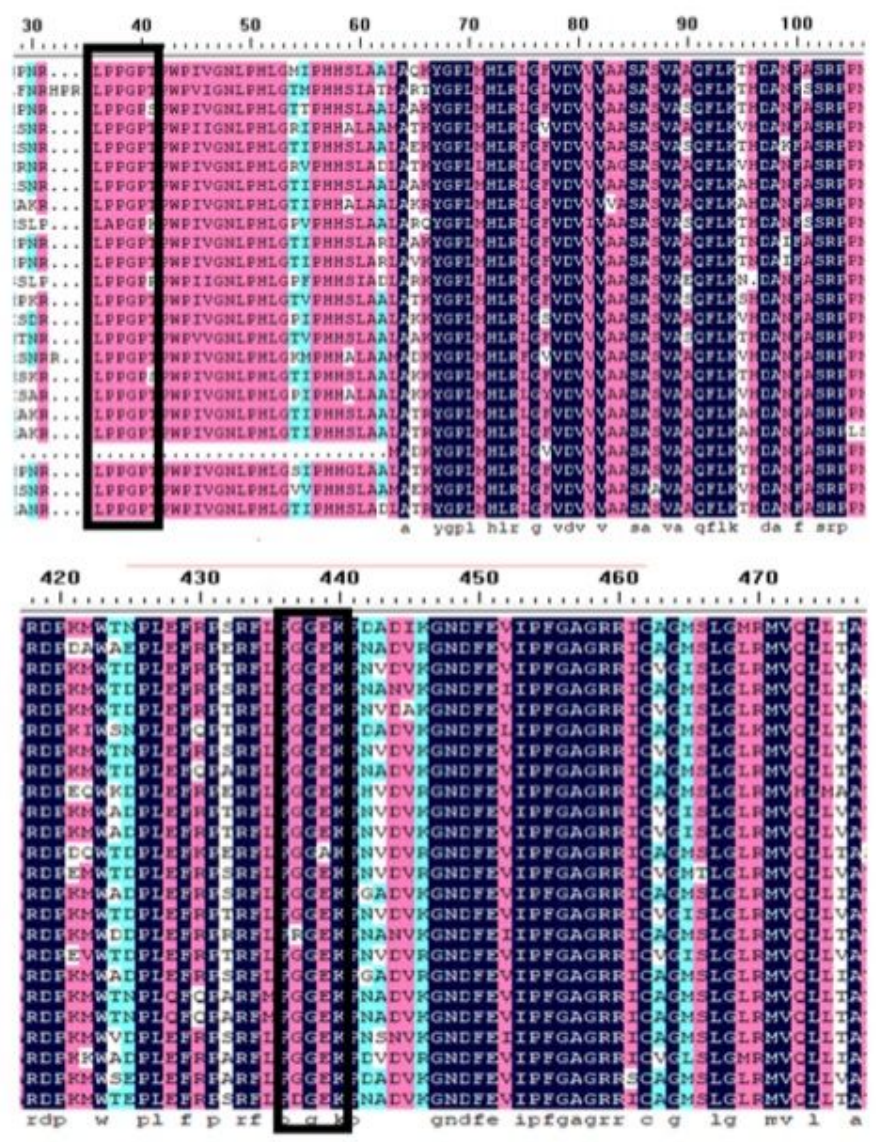

\section{Figure 6}

Molecular cloning and sequence analysis of the CtF3'H5 from safflower (A) PCR amplification of CtF3'H5 gene; (B) Multiple sequence alignment of F3'H amino acid obtained from safflower and other plant species; (C) Prediction of the tertiary structure of the protein encoded by the CtF3'H5. 


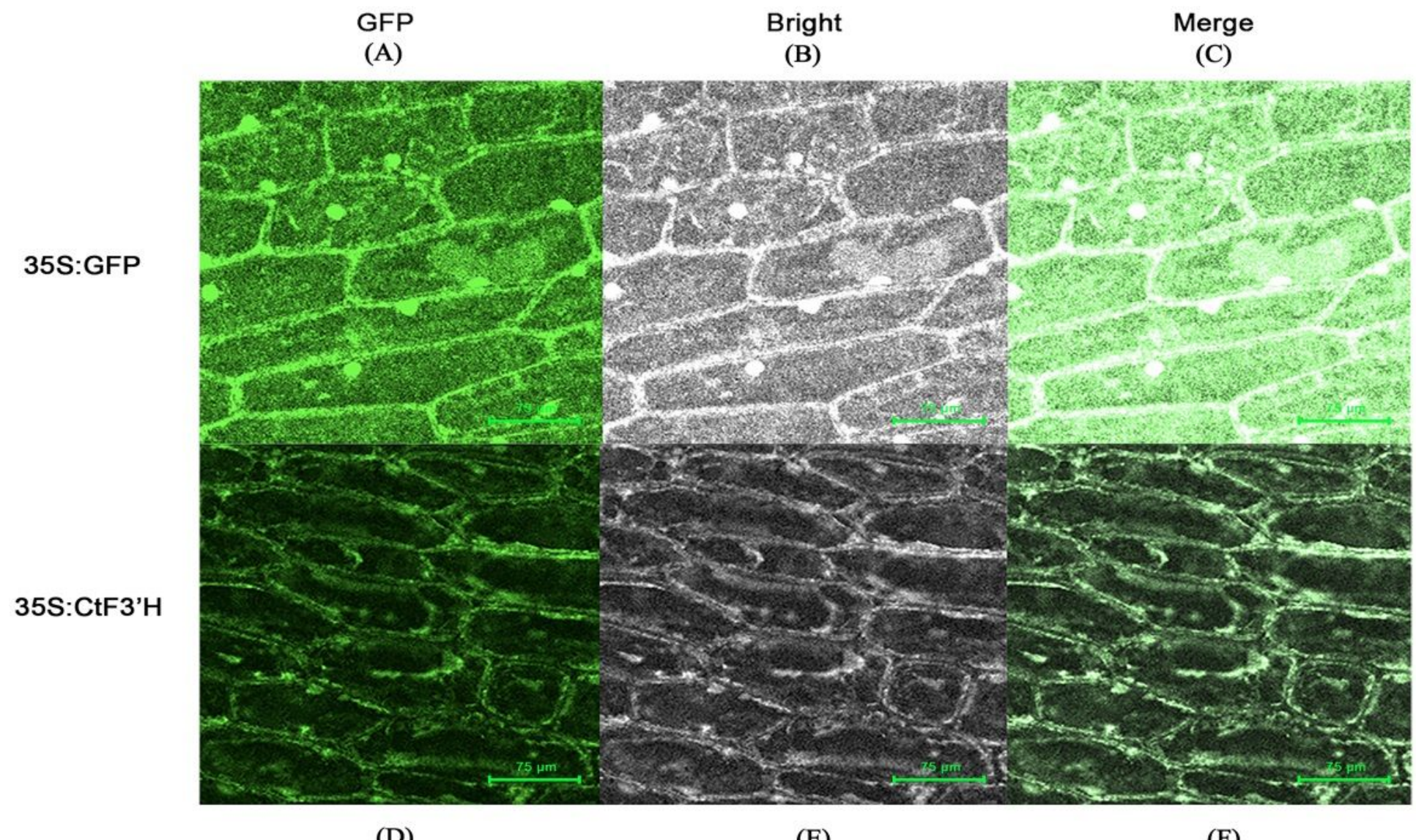

(D)

(E)

(F)

\section{Figure 7}

The GPF florescence pattern of CtF3'H5 under SEM. (A-C) The induction of empty vector 35S:GFP in onion cells. (D-F) The transient expression of pCAMBIA-CtF3'H5-GFP vector using the onion epidermal cells. Scar bar $=75 \mu \mathrm{m}$. 

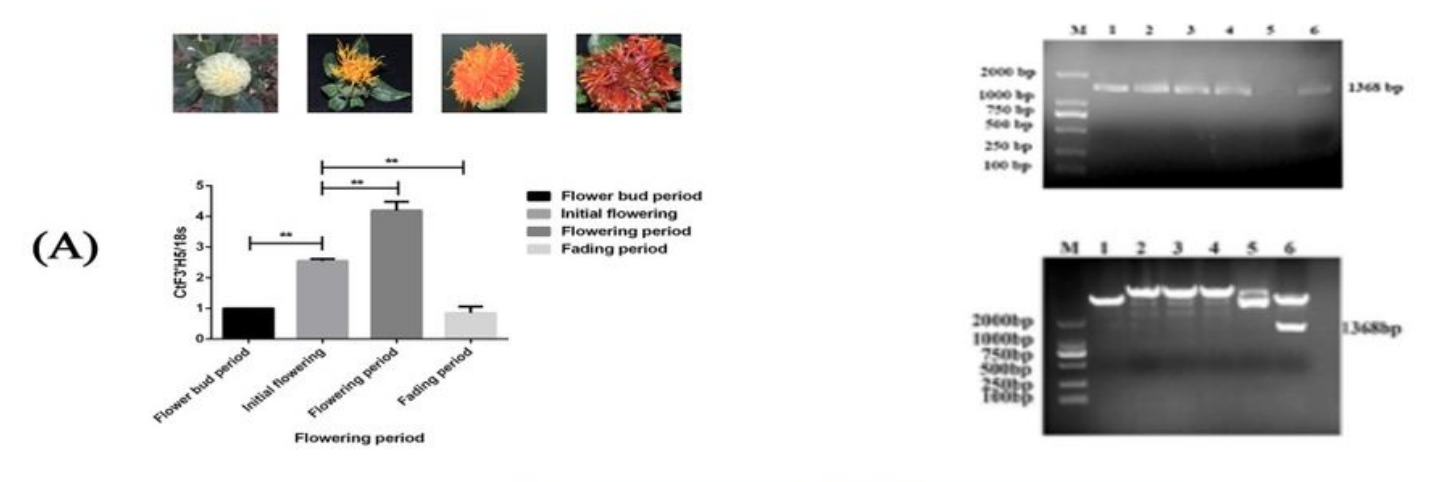

(B)

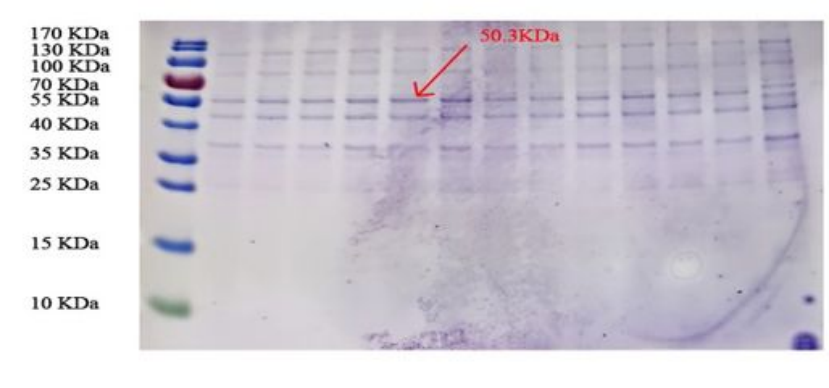

(D)

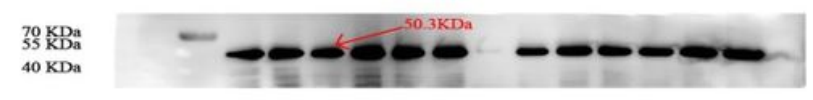

(E)

\section{Figure 8}

Expression analysis, heterologous expression and western blot hybridization of CtF3'H5 target protein from safflower (A) The realtime quantitative expression level of CtF3'H5 in four different flower developmental stages in safflower (B-C) Cloning and confirmation of PET28a+-CtF3'H5 recombinant plasmid using double restriction digestion sytem. (D) SDS-PAGE analysis of heterologously expressed CtF3'H5 protein under various concentration of 4 IPTG (E) Western Blot analysis of CtF3'H5 protein.The different concentrations of IPTG used in this experiment were as follow:L 0.2 IPTG; 0.4 IPTG, 0.5 IPTG, 0.6 IPTG, 0.7 IPTG, 0.8 IPTG.

\section{Supplementary Files}

This is a list of supplementary files associated with this preprint. Click to download.

- Supplementarymaterial.docx 